\title{
OS CUIDADOS PSICOLÓGICOS E DEPRESSÃO EM INDIVÍDUOS SUBMETIDOS A CIRURGIA BARIÁTRICA
}

\section{ARTIGO ORIGINAL}

SERRA, Janete Rodrigues ${ }^{1}$

BOTELHO, Helena Rubia De Santana ${ }^{2}$

SERRA, Janete Rodrigues. BOTELHO, Helena Rubia De Santana. Os cuidados psicológicos e depressão em indivíduos submetidos a cirurgia bariátrica. Revista Científica Multidisciplinar Núcleo do Conhecimento. Ano 05, Ed. 03, Vol. 05, pp. 102-129. Março de 2020. ISSN: 2448-0959, Link de acesso: https://www.nucleodoconhecimento.com.br/psicologia/cuidados-psicologicos

\section{RESUMO}

Esta pesquisa teve como objeto de estudo a depressão em pacientes que fizeram a cirurgia bariátrica; em alguns casos os pacientes tendem a demonstrar efeitos psicossociais após a cirurgia, sendo a depressão um dos mais comuns. O psicólogo, nesses casos, é de suma importância no pré e no pós-operatório do paciente. A relevância desta pesquisa se dá para que outras pesquisas possam contribuir com a compreensão do fenômeno, e, assim, contribuir para a elaboração de propostas de interesse em saúde pública. A pesquisa foi de abordagem qualitativa com revisão bibliográfica de estudo do tema, exploratória. Utilizou-se o método indutivo, buscando a constatação de dados. Para tanto, foram consultados os bancos de dados Scientific Eletronic Library Online (Scielo), Google Acadêmico compreendendo artigos com

1 Pós Graduação Em Psicologia Clínica, Graduação Em Psicologia (Bacharel), Tecnológica Em Recursos Humanos.

2 Doutorado em Saúde Publica. Mestrado em Ciências Da Saúde. Especialização em Gestão Em Unidades De Saúde. Especialização em Psicologia Hospitalar. Graduação em Psicologia. 
publicações referentes aos últimos 05 anos. Os resultados encontrados evidenciam a depressão e outros gravames. Como alguns resultados, pode-se explicitar o conhecimento de como os padrões impostos pela sociedade afetam diretamente a população obesa a ponto de investirem em procedimentos agressivos, a exemplo da cirurgia bariátrica e como o acompanhamento do psicólogo é importante para o autoconhecimento e reconhecimento da hora certa ou não para a cirurgia.

Palavras-chave: Cirurgia Bariátrica, depressão, psicólogo, aspectos psicossociais.

\section{INTRODUÇÃO}

O termo "depressão" conceitua-se de inúmeras formas. Enquanto termo pode-se conceituar como sintoma de transtorno de humor, transtorno de estresse e demais doenças; enquanto síndrome, a depressão pode incluir as variadas formas de alterações do humor, a exemplo da tristeza, apatia, desânimo, etc. Como doença a depressão se classifica como transtorno depressivo, melancolia, distimia, transtornos de bipolaridade, etc. (DEL PORTO, 1999). Ainda conforme o autor supracitado, o termo depressão é relativamente novo na História, entretanto, teve uso pela primeira vez em 1680, para definir um estado de desânimo, apatia e falta de interesse.

Relacionar os aspectos psicológicos que norteiam a cirurgia bariátrica e a depressão possibilita que os pacientes possam chegar à análise por caminhos diversos, buscando resolver questões práticas as mais variadas, investindo de diferentes maneiras e, a partir de pressupostos diversos, essa procura por tratamento e acompanhamento psicológico antes e depois da cirurgia. Desta forma, a presente pesquisa enseja-nos o questionamento: Qual a relação entre a cirurgia e a depressão?

A relevância da pesquisa se dá para que pesquisas possam contribuir com a compreensão do fenômeno, e, assim, contribuir para a elaboração de propostas de interesse em saúde pública. Espera-se, com este trabalho, contribuir para ampliar o conhecimento e compreensão dos aspectos psicológicos dessa doença, associada às dimensões em análise - depressão, que tem sido estudada, sobretudo, do ponto de vista médico, psicológico, mas ainda de forma incipiente, pela Psicologia, 
contribuindo, deste modo, para o aprofundamento dessa área e o desenvolvimento de futuros planos de intervenção.

Para alcançar esses dados foram elaborados objetivos que consistem em: a) investigar os fatores que envolvem o desencadeamento da depressão em pessoas submetidas à cirurgia bariátrica; b) descrever as implicações psicossociais em indivíduos submetidos à cirurgia bariátrica; c) demonstrar os índices de incidência, permanência ou agravamento da depressão, após a cirurgia; d) apontar a importância do acompanhamento psicológico frente ao processo da cirurgia bariátrica.

Recorreram-se os autores: Amaral e Pereira (2008), Dias et al. (2017), Sousa (2011) e outros. A pesquisa baseou-se no método bibliográfico e indutivo, com fontes entre os anos de 2008 e 2018, buscando-se expor o máximo de informações atualizadas a respeito do assunto.

O trabalho encontra-se estruturado em quatro tópicos principais. O primeiro refere-se à fundamentação teórica, na qual foram discutidos os principais conceitos que embasam a dissertação, entre eles os conceitos de depressão e a sua relação com a cirurgia bariátrica, tal como a atuação do psicólogo no processo de adaptação depois da cirurgia. $\mathrm{O}$ tópico seguinte traz o método, que destaca a pesquisa qualitativa $\mathrm{e}$ bibliográfica; por último, os resultados e discussões sobre os assuntos levantados no artigo.

\section{OBESIDADE E CIRURGIA BARIÁTRICA}

A obesidade é caracterizada pelo acúmulo em excesso de gordura corporal no indivíduo e um fator de risco para inúmeras doenças, entre elas, hipertensão, doenças cardiovasculares e diabetes, sendo considerada pela Organização Mundial de Saúde (OMS) como uma epidemia mundial que se origina principalmente pela má alimentação e pelo sedentarismo (DIAS et al., 2017).

O excesso de peso sempre tem acompanhado a Humanidade, sendo muitas vezes visto como sinônimo de saúde e de prosperidade, entretanto, hoje em dia, é 
considerado como uma doença crônica que afeta as pessoas independentemente de classe social, faixa etária ou gênero (SILVA, 2006).

A obesidade não é somente associada a um problema de saúde pública, mas a diversos processos biopsicossociais em que o "ambiente" (seja ele econômico, político seja ainda sociocultural) e não somente a pessoa em si, pois assume uma parte na análise do problema (DIAS et al., 2017).

Para ser diagnosticada é necessário um parâmetro que é comumente utilizado: o Índice de Massa Corporal (IMC). Este índice é calculado dividindo-se o peso do paciente pela sua altura elevada ao quadrado. Segundo a OMS, o padrão utilizado para o peso considerado normal ocorre quando este resultado é entre 18,5 e 24,9 . Para que um paciente seja diagnosticado obeso seu IMC deve estar acima de 30. Apesar de a obesidade coincidir com o aumento do peso, nem todo aumento de peso corresponde à obesidade. A Tabela 1 demonstra os diferentes níveis de obesidade para indivíduos adultos (OMS, 2004).

Tabela 1- Níveis de IMC

\begin{tabular}{|l|l|}
\hline Classificação & IMC (Kg/M2) \\
\hline Baixo Peso & Menor Que 18,5 \\
\hline Normal & $18,5-24,9$ \\
\hline Sobrepeso & Maior Que 25 \\
\hline Pré-Obeso & $25-29,9$ \\
\hline Obeso I & $30-34,9$ \\
\hline Obeso II & $35-39,9$ \\
\hline Obeso III & Maior Que 40 \\
\hline
\end{tabular}

Fonte: Quetelet (1832, apud OMS, 2004, p. 20).

O excesso de peso de um adulto tem como referência a sua altura, outrossim, a obesidade reflete a quantidade de tecido adiposo, embora as doenças relacionadas à obesidade estejam interligadas com a distribuição morfológica da gordura. Assim, 
então existem dois tipos principais: a obesidade ginoide (tipo pera), onde a gordura é de certa forma localizada principalmente nas coxas, ancas e nádegas, sendo esse tipo mais comum no sexo feminino (AMARAL; PEREIRA, 2008).

Já o segundo tipo é classificado como obesidade andróide (tipo maçã), onde a gordura é predominante na região abdominal, sendo, então, uma característica do sexo masculino (AMARAL; PEREIRA, 2008).

O IMC é um bom indicador, mas não totalmente correlacionado com a gordura corporal(B). As suas limitações são:

- não distingue massa gordurosa de massa magra, podendo ser pouco estimado em indivíduos mais velhos, em decorrência de sua perda de massa magra e diminuição do peso, e superestimado em indivíduos musculosos; não reflete, necessariamente, a distribuição da gordura corporal. A medida da distribuição de gordura é importante na avaliação de sobrepeso e obesidade porque a gordura visceral (intra-abdominal) é um fator de risco potencial para a doença, independentemente da gordura corporal total. Indivíduos com o mesmo IMC podem ter diferentes níveis de massa gordurosa visceral. Por exemplo: a distribuição de gordura abdominal é claramente influenciada pelo sexo: para algum acúmulo de gordura corporal, o homem tem, em média, o dobro da quantidade de gordura abdominal em relação à mulher na pré-menopausa; não indica necessariamente 0 mesmo grau de gordura em populações diversas, particularmente por causa das diferentes proporções corporais (ABESO, 2009, p 12).

Para os métodos que levam em consideração as formas antropomórficas, como o diâmetro da cintura são utilizadas as seguintes medidas: para o sexo masculino considera-se risco elevado quando a cintura encontra-se com o diâmetro de 94 a 102 
cm e maior que essa medida passa a ser considerado de alto risco. Já para o sexo feminino, o risco elevado encontra-se na faixa de 80 a $88 \mathrm{~cm}$; já o alto risco ocorre quando esse se encontra maior que $88 \mathrm{~cm}$ (AMARAL; PEREIRA, 2008).

Uns dos fatores que influenciam diretamente a obesidade são os biológicos e genéticos. Para Lent (2004 apud SOUSA et al., 2011),cada pessoa em sua particularidade está sujeita a vários fatores, entre eles os genéticos para escolher a quantidade de alimentos que vai consumir e este mesmo comportamento pode ter seus mecanismos variados de pessoa para pessoa, dependendo do estado emocional.

Segundo o autor supracitado, o hipotálamo (uma importante parte do cérebro humano fundamental como de controle da maior parte dos hormônios) tem duas áreas que regulam a ingestão de alimentos. São elas: a região lateral e a ventromedial.

A região lateral relaciona-se ao centro da fome, já a região ventromedial está ligada à saciedade. Todas as regiões do hipotálamo estão sob influência do sistema límbico e qualquer alteração desse sistema liga-se e interfere na vontade de comer. É a chamada fome.

No comportamento alimentar há outro fator importante que é o metabolismo, que se altera de acordo com a idade do indivíduo, este ao passar do tempo, ao ingerir a mesma quantidade de comida, vê os gastos irem diminuindo, fator este que também corrobora com a obesidade. A obesidade acontece quando é superior aos gastos energéticos, dirigidos pelo metabolismo.

O comportamento alimentar tem duas funções importantes: manter os nutrientes necessários para a sobrevivência do indivíduo (processos fisiológicos) e o prazer que 0 ato de comer proporciona, liberando neurotransmissores (serotonina e dopamina) responsáveis pelo prazer e bem-estar do indivíduo (LENT, 2004 apud SOUSA et al., 2011).

Os impactos da obesidade podem ser sentidos pelos indivíduos muito além das questões físicas, pois, em decorrência disso, tem-se uma menor qualidade de vida, 
mais acesso aos serviços de saúde, ausências no ambiente de trabalho e escolar, limitações nas atividades diárias e etc. (AMARAL; PEREIRA, 2008).

Por isso, muitas pessoas, que sofrem de obesidade, recorrem a vários procedimentos para conseguir perder peso, além de riscos de doenças. A estética e o enquadramento no padrão de beleza imposto por indústrias de cosméticos ensejam cada vez mais pessoas a se submeterem a métodos de emagrecimento, tais como a cirurgia bariátrica.

A cirurgia bariátrica, também conhecida como gastroplastia e cirurgia de redução de estômago, é um procedimento cirúrgico com o objetivo de proporcionar a perda de peso. O tratamento cirúrgico para a obesidade surgiu na década de 1950 , sendo que as técnicas foram aperfeiçoadas com o passar dos anos e, devido a essas melhorias, a cirurgia vem sendo bem mais aceita entre médicos e pacientes (SILVA, 2006).

Entretanto, a opção da cirurgia ocorre para pacientes que já esgotaram outros tipos de tratamento e não obtiveram sucesso. Um dos critérios para os candidatos à cirurgia bariátrica é a obesidade mórbida que está classifica pela tabela de Adophe Quetelet (1832), citada acima, com pessoas cujo IMC é maior ou igual a 40.

Como benefícios dessa cirurgia podem ser citados: a melhora acentuada de doenças crônicas, como a hipertensão, diabetes e hiperlipidemia, patologias que podem ser consequência do aumento excessivo de peso; entretanto, é importante ressaltar que somente a cirurgia não apresenta eficácia total no tratamento da obesidade, visto que é necessário que o paciente se comprometa em continuar o tratamento pós-cirurgia (BORDALO et al., 2010).

Já como risco desse tipo de processo cirúrgico, pode ser destacado a menor absorção de nutrientes, principalmente as proteínas e gordura. Então os nutrientes que necessitam da gordura, para serem absorvidos, como as vitaminas lipossolúveis e o zinco, são exemplos de vitaminas que podem ter uma má absorção durante esse tipo de procedimento (BORDALO et al., 2010). 
Vitaminas e minerais são fatores e cofatores essenciais em muitos processos biológicos que regulam o peso corporal direta ou indiretamente. Os benefícios metabólicos desses micronutrientes no controle da perda de peso incluem a regulação do apetite, da fome, da absorção de nutrientes, da taxa metabólica, do metabolismo de lipídios e carboidratos, das funções das glândulas tireoide e suprarrenais, do armazenamento de energia, da homeostase da glicose, de atividades neurais, entre outros. Assim, a "adequação" de micronutrientes é importante não só para a manutenção da saúde, mas também para obter o máximo sucesso na manutenção e na perda de peso em longo prazo (BORDALO et al., 2010, p. 114).

A resolução do Conselho Federal de Medicina (CFM) ํo 1.942/10 estabelece as normas de segurança para o tratamento da obesidade com tipo de procedimento cirúrgico, definindo as indicações, procedimentos e equipe técnica necessária para o tratamento. Embora a cirurgia bariátrica já seja um procedimento utilizado há décadas, as condições estruturais da pessoa submetida a esse tratamento são fundamentais, a equipe técnica deve ser composta de um cirurgião com formação específica na área, anestesiologista, clínico geral ou de especialidades relacionadas (endocrinologista, pneumologista), equipe de enfermagem especializada, fisioterapeuta, nutrólogo ou nutricionista, psiquiatra e/ou psicólogo (Instituto de Estudos de Saúde Suplementar) (IESS, 2018).

Há um arranjo da equipe técnica, no trabalho multidisciplinar para que as condições do paciente, antes da cirurgia, sejam favoráveis, diminuindo assim as possíveis implicações pós-cirurgia.

Embora haja essa preocupação com a multidisciplinaridade na preparação das pessoas que serão submetidas à cirurgia bariátrica, muitos pacientes encontram-se depositando todas as suas expectativas na mesa de cirurgia, onde começa a nascer uma crença excessiva num milagre, contudo, o que acontece na grande maioria dos casos é que, apesar do processo ser um sucesso, muitos pacientes voltam a 
recuperarem o peso perdido e isto pode trazer sérios problemas psicológicos (SILVA, 2006).

O que vem acontecendo com bastante recorrência são pacientes com graus moderados de obesidade que recorrem ao processo cirúrgico sem realmente haver necessidade dele, e, um dos principais motivos que enseja essas pessoas a procurarem esse tipo de cirurgia é para que haja um alívio de necessidades existenciais que se encontram conectados com os padrões de beleza impostos pela sociedade (SILVA, 2006).

A cirurgia bariátrica é importante para a diminuição de peso de forma mais rápida, já que, no procedimento cirúrgico, há redução do tamanho do estômago. Essa prática desencadeia algumas implicações nos indivíduos e determinados fatores não cirúrgicos e psicológicos causam influências na capacidade do paciente a ajustar-se a novas realidades, condições e estilos de vida.

Alguns pacientes, mesmo após a cirurgia, continuam com o objetivo na perda do peso e isto pode influenciar na sua imagem corporal; outros, na busca da satisfação corporal, criam expectativas que podem colaborar de forma negativa no seu comportamento, desencadeando ansiedade e insatisfação.

\subsection{IMPLICAÇÕES PSICOSSOCIAIS EM PACIENTES SUBMETIDOS À CIRURGIA BARIÁTRICA-DEPRESSÃO}

Segundo pesquisas, a destacar as da Universidade de São Paulo (USP), do Programa de Atenção aos Transtornos Alimentares (PROATA) e da Universidade Federal de São Paulo (UNIFESP), vários estudos, feitos nos últimos anos apontam que cada vez mais pacientes que se submetem ao procedimento cirúrgico para perda de peso, têm apresentado comportamentos compulsivos, depressão e ansiedade, e em casos extremos, chegam a cometer suicídio, ainda assim estando magro como o desejado (PROATA, 2010). 
Leal e Baldin (2007) explicam que muitas das vezes o emagrecimento súbito incorre em problemas como sintomas de ansiedade, depressão, ideação suicida entre outros; desta forma, é possível analisar que as fantasias, a expectativa, as frustrações, os resultados, são situações enfrentadas antes e depois do procedimento cirúrgico e que podem estar associados a expectativas de padrões de beleza impostos pela sociedade.

Um dos problemas que pode se manifestar após a cirurgia bariátrica é a depressão, que é definida pela OMS (2004) como um "transtorno mental" comum, que afeta milhões de pessoas em todo o mundo. Entretanto, até mesmo por conta de levantamentos e apresentações de estatísticas como essas, o problema das depressões nos dias atuais destaca a atenção principalmente por conta da dimensão, do papel e da imagem que tal problemática assume na atualidade.

A subjetividade dos pacientes que se submetem à cirurgia bariátrica não pode ser ignorada, uma vez que nem sempre o enfretamento da complexidade da cirurgia é satisfatório; outrossim, a maturidade desse paciente e sua personalidade podem ser um complicador a mais e fator que gera ansiedade e depressão.

A depressão é vista como um mito em saúde mental na atualidade. Estatísticas de levantamentos realizados pela OMS (2004) consideram que a depressão colabora como uma das principais causas para afastamento do trabalho, incapacitando os indivíduos de exercer suas atividades laborais, vivenciar sua existência nas relações sociais, isto devido à introspecção e ao isolamento que estes estados afetivos implicam.

A Psicologia colabora com o reconhecimento dos benefícios que a perda de peso pode trazer ao paciente, mas vê com menos entusiasmo a questão do emagrecimento súbito e acentuado como resultado de técnicas cirúrgicas. A obesidade funciona muitas vezes como um falso "selfie" mental e corporal, que protege um verdadeiro eu fragilizado e mal estruturado, atribuindo-se a isso a enormes dificuldades no processo de emagrecimento; para tais fatores defende-se a importância da assistência psicológica antes e depois da cirurgia. 
Como a questão de estética também é fator determinante para a decisão de se submeter à cirurgia bariátrica, a promoção de mudanças que este procedimento oferece na imagem corporal do paciente, de maneira súbita, colabora com as alterações psicológicas, que ocorrem não na mesma rapidez que o resultado da cirurgia, favorecendo que o paciente, mesmo com a perda significativa ou esperada do peso, se veja ainda como obeso (BASTOS et al.,2013).

Cabe ressaltar que o paciente pode enfrentar com a visão ainda da obesidade no corpo magro, sentimentos diversos, tais como de arrependimentos, medo, estresse pela ansiedade na busca do corpo perfeito, sentimento de raiva pela reeducação alimentar, dentre outros comportamentos que implicam em sofrimento psíquico.

\subsection{A IMPORTÂNCIA DA ASSISTÊNCIA PSICOLÓGICA FRENTE À CIRURGIA BARIÁTRICA}

O profissional de Psicologia é parte integrante da equipe técnica pré-cirúrgica para o procedimento bariátrico, entretanto seu papel vai além de avaliação psicológica que habilita os pacientes para o a cirurgia. Frente às dificuldades que os pacientes enfrentam no processo pré e pós-operatório, colhe-se a necessidade da intervenção do psicólogo nesse contexto e a importância de seu papel tendo em vista obesidade e a cirurgia bariátrica.

Apesar de a avaliação psicológica fazer parte do pré-operatório, ela não deve ser baseada somente em reconhecer transtornos mentais atuais e preexistentes. $\mathrm{O}$ acompanhamento desse paciente deve ser feito também no pós-operatório, com intervalos regulares, visando também melhorias no psicológico após a cirurgia (FANDIÑO et al., 2004).

A avaliação psicológica ajuda a reconhecer os sintomas da depressão e aqueles ligados às mudanças de humor e transtornos alimentares, geralmente em pacientes com obesidade grau III que já fazem tratamentos para emagrecer. Devido ao aparecimento recorrente desses sintomas, o psicólogo precisa estar preparado para reconhecer sinais que indiquem um episódio depressivo que venha a ocorrer 
novamente durante o pós-operatório. Esses episódios necessitam de atenção para que, em determinados casos, não levem ao suicídio (FANDIÑO et al., 2004).

Para os autores supracitados, é interessante observar que geralmente a obesidade encontra-se ligada a outros problemas independentes dos aspectos biológicos presentes. Como exemplo tem-se a Compulsão Alimentar Periódica (CAP), quando o indivíduo ingere, em um período limitado de tempo, uma grande quantidade de alimentos; na maioria das vezes essa quantidade de alimentos é maior do que outras pessoas consumiriam no mesmo período, além de virem acompanhadas por falta de controle sobre o comportamento alimentar.

O profissional de Psicologia deve estar preparado para utilizar diferentes formas de trabalho com pacientes pré-cirúrgicos. Vicentin e Terzis (2011) concluem que a forma mais utilizada por psicólogos é a técnica grupal, que é um método de aplicação de técnicas terapêuticas que funcionam como instrumento de mudança e cura a grupos de pacientes.

A técnica em grupo favorece a um maior número de pessoas, contribui para a parte menos favorecidas de pessoas tenha acesso à psicoterapia por esta modalidade de atendimento, ser mais em conta que um atendimento individual (VICENTIN; TERZIS, 2011). Outros psicólogos preferem atuar de forma individual com o paciente e possibilitam ao familiar a inclusão no processo de preparação às mudanças existentes. Já Stuart (1999) afirma que a família tem seu papel fundamental no auxílio das mudanças de comportamentos dos pacientes obesos, encaminhando-os a melhores hábitos alimentares.

O psicólogo, independentemente da forma de abordagem com o cliente, exerce em seu papel um importante trabalho que é preparar os pacientes para o processo cirúrgico e orientá-los às mudanças e à relevância de um planejamento alimentar após o procedimento cirúrgico, diminuindo assim os impactos psicossociais e condicionando o paciente ao melhor enfrentamento de todo o processo que envolve 0 tratamento da obesidade junto à cirurgia bariátrica. 
É relevante ressaltar que o trabalho do psicólogo vai além da avaliação psicológica e do acompanhamento pós-cirúrgicos, pois diversos fatores emocionais podem implicar em transtornos do humor, ansiedade, depressão que podem afetar o condicionamento do paciente para enfrentar as mudanças em sua vida após a cirurgia, portanto a importância da assistência psicológica infere na qualidade de vida do paciente que pode vivenciar da melhor forma possível, o resultado de suas escolhas.

O trabalho do psicólogo com os pacientes bariátricos é importante sob vários aspectos e entre eles a avaliação psicológica e o acompanhamento. Na avaliação psicológica o psicólogo pode identificar nos pacientes as tendências de comportamentos que vão norteá-los para elaboração de um laudo psicológico que comprove a necessidade da cirurgia e suas implicações na vida do paciente, cabe ressaltar que o papel do psicólogo nesse contexto é imprescindível. No acompanhamento psicológico o profissional da psicologia atua na prevenção e no tratamento dos problemas que surgiram no pré ou pós-operatório, uma vez que se submeter à cirurgia bariátrica pode não ser a solução dos problemas do paciente, sendo que este precisa estar preparado para vivenciar as mudanças que essa escolha vai causar em sua vida.

\section{MÉTODO}

A pesquisa presente é caracterizada como bibliográfica, utilizando-se como materiais e fontes de pesquisa livros, artigos, monografias e reportagens sobre o assunto dos últimos dez anos. Foram levantados também alguns dados quantitativos em órgãos como a OMS e o Conselho Regional de Medicina.

A pesquisa bibliográfica teve como intuito fazer um apanhado geral sobre o material já escrito a respeito do assunto, pois esses materiais oferecem dados atualizados e referentes ao tema discutido, evitando-se assim a repetição de erros (LAKATOS; MARCONI, 2017).

Para iniciar a pesquisa levou-se em consideração um apanhado primeiramente histórico e conceitual a respeito da cirurgia bariátrica e da depressão, ambos os 
objetos de estudo deste trabalho, buscando-se definir as melhores fontes documentais que melhor se encaixassem no eixo da pesquisa.

Segundo Lakatos e Marconi (2017), antes de iniciar-se uma pesquisa, cumpre analisar essas fontes documentais, fazendo uma investigação preliminar que deve ser realizada a partir da análise de documentos. Em virtude da necessidade de ampliação nos estudos sobre as implicações psicossociais em pacientes bariátricos, há necessidade de abordar o indivíduo com obesidade e compreender seus aspectos psicológicos frente à cirurgia bariátrica. O processo de levantamento de dados surgiu com orientação de pesquisa de revisão bibliográfica exploratória e qualitativa, artigos científicos e livros.

A pesquisa exploratória responde às diversas questões, pois se realiza o estudo, a análise, o registro e a interpretação dos fatos sem a interferência do pesquisador. Segundo Campos (2015), as pesquisas documentais possuem fases determinadas: Identificação do material documental existente, localização desse material, recuperação do material, leitura e compilação e fichamento dos documentos e materiais analisados.

A pesquisa qualitativa se baseia no caráter subjetivo do objeto analisado. Nesse tipo de pesquisa os entrevistados se encontram mais livres para expressar seus pontos de vista sobre os assuntos pesquisados e as pesquisas não têm o intuito de contabilizar dados, mas sim de obter respostas que demonstrem determinados comportamentos em um grupo-alvo.

Por último, utilizou-se o método indutivo, buscando-se a constatação de dados, e visando-se concluir uma análise da relação da cirurgia bariátrica com a depressão. $\mathrm{O}$ método indutivo parte de três pontos, segundo Lakatos e Marconi (2017). O primeiro, a observação do fenômeno; neste caso, os sintomas psicossociais pós-cirurgia bariátrica; a relação entre eles também se encontra presente ao presumir que existem sintomas negativos decorrente da cirurgia; o terceiro ponto é a generalização da relação onde se tem a relação entre os fatos e fenômenos semelhantes. 


\section{RESULTADOS E DISCUSSÕES}

Procurou-se inicialmente entender o conceito da depressão, seus sintomas e suas consequências, como também o conceito de obesidade, assim como os números relevantes à questão como os utilizados para estabelecer o IMC e os dados mundiais a respeito da obesidade no Brasil e no mundo. A organização dos dados levantados ocorreu através da pesquisa bibliográfica que estivesse relacionada com a depressão e a cirurgia bariátrica, esse resultado foi obtido através de pesquisas que já tratavam sobre a depressão e também sobre a obesidade e a cirurgia, fundamentando assim este trabalho. $\mathrm{O}$ objetivo foi relacionar o desencadeamento da depressão em pessoas submetidas à cirurgia bariátrica, descrever as implicações psicossociais advindas desta, que envolvem o sofrimento psíquico frente à cirurgia bariátrica e apontar o papel do psicólogo no pré e pós-operatório.

A principal forma de levantar estes resultados se deu através da pesquisa bibliográfica, formulação de banco de dados e da análise de trabalhos previamente publicados, também a comparação do período de publicações, as buscas por mais literaturas, onde se observou a necessidade de mais estudos sobre o assunto, o achado mais recente deu-se os anos de 2012 e 2018.

A pesquisa ofereceu a possibilidade do reconhecimento de alguns sintomas característicos da depressão, assim como seus efeitos, como a baixa autoestima e transtornos alimentares, além das consequências psicossociais presentes e juntamente com as implicações fisiológicas. Dessa forma, buscou-se, neste trabalho, explorar as implicações psicológicas na vida do paciente bariátrico.

Através da pesquisa, foi possível compreender que tanto as técnicas em grupo e as individuais de terapia ajudam o indivíduo a se preparar melhor para o procedimento; por isso, a participação do psicólogo é de fundamental importância desde a tomada de decisão de fazer a cirurgia até o pós-cirúrgico, respondendo ao objetivo desta pesquisa de investigar o papel do psicólogo quanto à cirurgia bariátrica. 
O papel do psicólogo no pré e pós-operatório são de suma importância, pois, ajuda o indivíduo a compreender não somente os sintomas que podem aparecer no decorrer do processo cirúrgico, quanto também os motivos que ensejam o indivíduo a fazer a cirurgia. A Psicologia, através desta pesquisa, contribui para favorecer as novas discussões sobre o papel do psicólogo no tratamento de pessoas obesas que se submeteram ou se submeterão a um processo cirúrgico. O psicólogo atua na orientação, prevenção, acolhimento, intervenção, assim favorecendo de maneira positiva ao enfrentamento da depressão, foco deste estudo.

Buscou-se entender a depressão e seus aspectos e impactos na vida do paciente bariátrico, com essa contribuição buscam-se novos aprofundamentos científicos na investigação sobre a depressão e a cirurgia bariátrica, o aprimoramento do profissional de psicologia que ampliará seus conhecimentos, elevando-se a novos questionamentos e respostas sobre a depressão.

O psicólogo precisa estar atento às técnicas utilizadas com os indivíduos em processo operatório, pois cada pessoa reage de uma forma diferente e é necessário adequar o tratamento às orientações em cada caso, prezando-se o bem-estar do paciente. Por meio de técnicas de grupo, ou de formas de tratamento dos possíveis sintomas que venham a aparecer, ou até mesmo em caso de prevenção do mau uso da cirurgia bariátrica, o psicólogo deve atuar variando as técnicas, o profissional pode fazer o acompanhamento tanto com o indivíduo quanto com a sua família e com as pessoas que convivem com o mesmo.

Conforme Pinto (2004), em relação ao papel do psicólogo no processo da cirurgia bariátrica, este classifica o trabalho do psicólogo em dois pontos: o primeiro na avaliação psicológica e o segundo se refere ao acompanhamento. A avaliação facilita o diagnóstico de transtornos alimentares, tendências compulsivas e a partir disso trabalhar em um laudo psicológico que comprove a necessidade da intervenção cirúrgica. O laudo também possibilita aos médicos o conhecimento de que o paciente está saudável e apto para o procedimento. 
O acompanhamento, por sua vez, é importante em casos em que a obesidade não tem as causas sanadas, pois os problemas podem aparecer em outros pontos da vida. O pré-operatório depende de como o paciente encara a situação, ansiedade e medo são reações normais, mas, quando se elevam e coincidem com outros aspectos, poderão causar consequências negativas no organismo do paciente (PINTO, 2004).

O apoio do psicólogo, junto às equipes multidisciplinares que fazem a cirurgia, deve persistir desde o pré-operatório dando continuidade no pós-operatório. Pode ser fundamentalmente clínica ou auxiliada por testes clínicos e através de entrevistas psiquiátricas estruturadas. Para que haja sucesso no processo pré-operatório o paciente deve dispor-se também a participar de tratamentos em longo prazo. O laudo psicológico é importante para um bom resultado da cirurgia e deve ser feito por profissionais capacitados, visando-se os melhores objetivos.

Como principais tópicos que podem ser destacados para esta discussão têm-se os padrões sociais que são impostos às pessoas que acabam por levar à cirurgia bariátrica muitas vezes quando realmente não há necessidade, para atender a fins puramente estéticos e não que estejam ligados à saúde do indivíduo, como os dados abaixo demonstram.

No Brasil, em 2015, mais de 100 mil cirurgias foram feitas tornando-se assim o segundo país em cirurgias bariátricas, ficando atrás do EUA, onde a cada ano esses números dobram. As cirurgias aumentam de acordo com o aumento da população obesa no país (MENDES, 2016).

Entretanto, mesmo com as taxas altas a cirurgia bariátrica não é um resultado definitivo para a obesidade, pois na maioria dos casos pode reincidir em menos de um ano e meio após a cirurgia, principalmente quando as causas não estão ligadas somente aos aspectos físicos, mas também a aspectos psicossociais como a ansiedade, a depressão e a baixa autoestima.

Um dos objetivos desta pesquisa foi o de estabelecer os fatores que desencadeiam a depressão em pessoas que se submeteram à cirurgia bariátrica, além dos motivos de 
saúde. Um dos principais pontos que foram evidenciados com este tratamento é de que os padrões de beleza impostos pela sociedade contribuem muito para como a pessoa se percebe no meio em que se encontra, pois muitas das pessoas que se submetem a esse processo fazem-no com o intuito de ficar magras para o resto da vida, o que não acontece com metade dos pacientes que fazem a cirurgia.

Em conformidade com os achados desta pesquisa, em uma reportagem para o site do G1 (2012), no programa Bem-Estar foi visto que metade das pessoas obesas, que faz a cirurgia, ganha peso parcialmente e cerca de $5 \%$ ganham todo o peso novamente. Isso se deve ao fato de as pessoas acharem que somente a cirurgia é o suficiente para a perda de peso, quando as maiorias dos cirurgiões bariátricos recomendam uma alimentação saudável e a prática de exercícios físicos, principalmente os aeróbicos pelo menos três vezes na semana.

Identificou-se nos resultados desta pesquisa, que o procedimento é visto como a última solução para o problema da obesidade, pois essa cirurgia não interfere somente no sistema gastrointestinal, mas também na absorção de vitaminas e sais minerais e também outras funções do corpo do paciente.

Cumpre ressaltar outra questão no processo cirúrgico da bariátrica, a psicológica, a qual é extremamente relevante, pois nesta pesquisa abordaram-se as implicações psicológicas que vão desde irritabilidade, ansiedade, compulsão e depressão. Abordou-se também a imagem corporal e sua relação com o adoecimento psicológico, como a depressão por exemplo.

Outro ponto importante na pesquisa refere-se aos achados de Silva (2006) que nos diz sobre duas formas de abordar o indivíduo que faz a cirurgia bariátrica. O primeiro se refere à Psicologia cognitivista, onde haveria um indivíduo em desequilíbrio com o próprio corpo e este busca adaptar-se às condições do meio. Seu corpo conta, então, com programações específicas. Assim sendo, o indivíduo obeso come como se tivesse automedicando-se e busca assim compensar alguns sintomas psicológicos como a ansiedade e o Transtorno do Comer Compulsivo (TCC), buscando um equilíbrio cerebral, para, por sua vez, conseguir um equilíbrio emocional. A segunda 
vertente proposta pela autora é a Psicanálise. Tem-se o questionamento do que causou esse tipo de necessidade, ou seja: o que motivou o paciente a utilizar a comida como forma de refúgio para os problemas psicológicos.

A partir dessa concepção pode-se refletir que comer de forma compulsiva não é somente uma questão biológica e adaptativa, mas também ligada ao subjetivo, considerando-se os aspectos culturais que formam a esfera humana. O papel do psicólogo aqui seria justamente o de ajudar o paciente a entender os outros aspectos do transtorno alimentar que enseja a obesidade e, consequentemente, a cirurgia bariátrica.

\section{CONSIDERAÇÕES FINAIS}

A pesquisa feita para o levantamento de dados deste estudo contribuiu para a minha formação como psicóloga, pois ajudou a agregar conhecimentos sobre a depressão, padrões sociais de beleza, assim como sua relação com a cirurgia bariátrica que foi observada tanto no contexto biológico quanto nos psicossociais.

Buscou-se relacionar os efeitos da cirurgia bariátrica com a depressão, assim como a intervenção do psicólogo no pré e pós-operatório do paciente, atentando-se para a necessidade de um laudo que permita o procedimento e um acompanhamento dos mesmos depois da cirurgia, visando-se um bem-estar com o novo corpo e com as necessidades do pós-operatório.

Para alguns autores referidos na pesquisa, o emagrecimento súbito poderá ensejar desordem emocional, sofrimento psíquico, a exemplo da depressão, ansiedade, ideação suicida, dentre outros. Desta forma, perceberam-se nos achados deste estudo que a cirurgia bariátrica gera implicações psicossociais à pessoa submetida a este procedimento. Percebeu-se ainda a importância da assistência psicológica durante todo o processo.

Os dados apresentados e discutidos no presente trabalho dissertativo permitem apontar que pode ocorrer relação no desencadeamento da depressão e a cirurgia 
bariátrica; entretanto, para os futuros estudos, recomenda-se o aprofundamento da temática, através do levantamento de dados por meio de entrevistas e questionários com pessoas que tenham passado pelo procedimento e que possam a querer contribuir para a compreensão da cirurgia bariátrica e suas implicações psicológicas na vida do indivíduo.

\section{REFERÊNCIAS}

AMARAL, O.; PEREIRA, C. Obesidade da genética ao ambiente. Revista Millenium, no 34, abril de $2008 . \quad$ Disponível em: $<$ https://revistas.rcaap.pt/millenium/article/view/8374>. Acesso em: 29 out. 2018.

ASSOCIAÇÃO BRASILEIRA PARA O ESTUDO DA OBESIDADE E DA SÍNDROME METABÓLICA - ABESO. Diretrizes Brasileiras de Obesidade 2009/2010. $3^{\text {a }}$ ed. Itapevi - SP, 2009.

BEM ESTAR. Metade dos obesos que reduzem o estômago volta a engordar. G1, 2012. Disponível em: <http://g1.globo.com/bemestar/noticia/2012/02/metade-dosobesos-que-reduzem-o-estomago-volta-engordar.html>. Acesso em: 27 out. 2018.

BASTOS et al. Fatores determinantes do reganho ponderal no pós-operatório de cirurgia bariátrica. $A B C D[o n l i n e] . \quad 2013$, vol.26. Disponível em: $<$ http://dx.doi.org/10.1590/S0102-67202013000600007.>. Acesso em: 06 de nov. de 2018

BORDALO, L. A. et al. Cirurgia bariátrica: como e por que suplementar. Viçosa - MG: Universidade Federal de Viçosa, 2010.

CAMPOS, L. F. L. Métodos e Técnicas de Pesquisa em Psicologia. 5ª Ed. Campinas: Alínea, 2015.

DEL PORTO, J. A. Conceito e diagnóstico. Revista Brasileira de Psiquiatria, São Paulo, $\quad$ v. $21, \quad 1999 . \quad$ Disponível em: <http://www.scielo.br/pdf/rbp/v21s1/v21s1a03.pdf>. Acesso: 15 out. 2018. 
DIAS, P. C. et.al. Obesidade e políticas públicas: concepções e estratégias adotadas pelo governo brasileiro. Caderno de Saúde Pública, Niterói, v. 33, 2017. Disponível em: $\quad<$ http://www.scielo.br/pdf/csp/v33n7/1678-4464-csp-33-07-e00006016.pdf>. Acesso em: 15 out. 2018.

FANDIÑO, J. et. al. Cirurgia Bariátrica: aspectos clínico-cirúrgicos e psiquiátricos. Revista de Psiquiatria do Rio Grande do Sul, v. 26, № 01, 2004. Disponível em: <http://www.scielo.br/scielo.php?pid=S0101$81082004000100007 \&$ script=sci_abstract\&tlng=pt>. Acesso em: 15 out. 2018.

INSTITUTO DE ESTUDOS DE SAÚDE SUPLEMENTAR - IESS. Cirurgia Bariátrica: Pareceres Técnico-Científicos. Vol 1. 2018. Disponível em: $<$ https://iess.org.br/?p=publicacoes\&id_tipo=7>. Acesso em: 15 out. 2018.

LAKATOS, E. M.; MARCONI, M. A. Metodologia do Trabalho Científico. 8. ed. São Paulo: Atlas, 2017.

LEAL, C. W.; BALDIN, N.O impacto emocional da cirurgia bariátrica em pacientes com obesidade mórbida.Revista de Psiquiatria do Rio Grande do Sul, Porto Alegre,vol.29, no $3,2007.2$ Disponível em: $<$ http://www.scielo.br/scielo.php?script=sci_arttext\&pid=S0101$81082007000300013>$. Acesso em: 27 abr. 2018.

MENDES, S. Cirurgia bariátrica: dez anos depois, menos da metade dos pacientes mantêm o peso. RFI. 2016. Disponível em:<http://br.rfi.fr/ciencias/20161004-cirurgiabariatrica-dez-anos-depois-menos-da-metade-dos-pacientes-mantem-o-peso>. Acesso em: 29 out. 2018.

Organização Mundial da Saúde - OMS. Obesidade: prevenindo e controlando a epidemia global: relatório da consultoria da OMS. São Paulo: Editora Roca; 2004.

PINTO, J. Cirurgias definitivas exigem acompanhamento terapêutico: médicos pedem laudos psicológicos para pacientes que se submetem a mudança de sexo e redução de estômago, $2004 . \quad$ Disponível 
em:<http://www.crpsp.org.br/portal/comunicacao/jornal_crp/141/frames/fr_conversan do_psicologo.aspx>. Acesso em: 29 out. 2018.

Programa de Atenção aos Transtornos Alimentares (PROATA). Unifesp. 2010. Disponível em: $<$ http://www.dpsiq.sites.unifesp.br/d/proata/ensino/pesquisa/index.html>. Acesso em: 06 de nov. 2018

SILVA, A. A. A. Cirurgia Bariátrica: como o psiquismo significa esse corte no corpo? Brasília: PUC - Brasília, 2006.

SOUSA, A. S. et al. O comportamento alimentar e os distúrbios psicológicos. 2011. 11 p. Trabalho de Curso (Psicologia)- Universidade Vila Velha, [S.I.], 2011.

STUART, R. B. Pense Magro e emagreça. Rio de janeiro: Record, 1999.

VICENTIN, A.; TERZIS, A. Psicoterapia de Grupo: construções teóricas. In: Encontro de Iniciação em Desenvolvimento Tecnológico e Inovação da PUC. Anais do XVI Encontro de Iniciação Científica. São Paulo: PUC, 2011.

Enviado: Agosto, 2019.

Aprovado: Março, 2020. 